This is the accepted version of the following article: [Jiangtao Sun and Wuqiang Yang, Fringe effect of electrical capacitance and resistance tomography sensors, Meas. Sci. and Technol., 24 (7), 2013, 074002 (15pp)], which has been published in final form at [doi:10.1088/0957-0233/24/7/074002]. In addition, authors may also transmit, print and share copies with colleagues, provided that there is no systematic distribution of the submitted version, e.g. posting on a listserve, network or automated delivery.

\title{
Fringe effect of electrical capacitance and resistance tomography sensors
}

\author{
Jiangtao Sun and Wuqiang Yang \\ School of Electrical and Electronic Engineering, The University of Manchester \\ Manchester M13 9PL, UK, e-mail: jiangtao.sun@postgrad.manchester.ac.uk
}

\begin{abstract}
Because of the "soft-field" nature, all electrical tomography sensors suffer from field distortion or 3D effect. In electrical resistance tomography (ERT) sensors, small pin electrodes are commonly used. It is well known that the pin electrodes result in severe field distortion or $3 \mathrm{D}$ effect, and the sensing region of such an ERT sensor is not constrained to the pin electrode plane, but spreads to a large volume. This is also true for electrical capacitance tomography (ECT) sensors, even though it is less severe because of larger electrodes and grounded end guards used. However, when the length of electrodes in an ECT sensor without guards is reduced to almost the same dimension as those in ERT sensor, the 3D effect may be equally obvious. To investigate the 3D effect of ERT and ECT sensors with and without guards, simulations were carried out with different lengths of electrodes and the results are compared with the corresponding $2 \mathrm{D}$ simulation. It is concluded that sensors with longer electrodes have less $3 \mathrm{D}$ effect. Because grounded end guards are effective in reducing the 3D effect of ECT sensors, we propose to apply grounded guards in ERT sensors and integrate ECT and ERT sensors together. While commonly current excitation is used with ERT sensors, we propose voltage excitation instead, the feasibility of which has been verified by experiment. Simulation results reveal that ERT sensors with grounded guards have less 3D effect. Finally, a common structure for reducing the 3D effect is proposed for ECT and ERT sensors for the first time, to simplify the sensor structure and reduce the number of connections for ECT/ERT dual-modality measurements.
\end{abstract}

Keywords- ECT sensor, ERT sensor, 3D effect, grounded guards, dual-modality

\section{INTRODUCTION}

According to the electrical parameter to be measured, electrical tomography (ET) can be electrical resistance tomography (ERT), electrical capacitance tomography (ECT) and electro-magnetic tomography (EMT). Unlike x-rays, which pass through a cross section along a straight path, ET is based on "soft-field" sensing, i.e. the electric field distribution depends on the material distribution, and the electric filed spreads to a large volume at both ends of the sensor. This field distortion at both ends of the sensor is referred to as 3D effect. In ERT sensors, small pin electrodes are commonly used for current injection excitation. It is well known that the pin electrodes result in severe field distortion or 3D effect, and the sensing region of such an ERT sensor is not constrained to the pin electrode plane, but spreads to a large volume. In the past, some researchers reported serious fringe effect with ERT sensors [1] [2], which would result in distortion of reconstructed images. Some other researchers made comparison between simulated electric fields of a 3D ERT sensor model and the corresponding 2D analysis by neglecting the fringe effect, or between 3D simulation and experimental results. They concluded that the simplified 2D model cannot be used to approximate the 3D field because of too large errors [3] [4].

To investigate the $3 \mathrm{D}$ effect on the measurements acquired from different electrode pairs, equi-current perturbation hypothesis was used in [5] to describe the image-forming mechanism of an electrical impedance tomography (EIT) system with an object extended from the electrode plane to a distant position in the axial direction of the sensor (Note that ERT is sometimes called EIT). It was pointed out that the largest change in the reconstructed image would take place 
where the disturbed equi-current surface with the largest current density intersects the cross-section of the EIT sensor. A circular image would be reconstructed if the extended objects cover the whole cross-section, but this becomes more complicated when the extended objects do not cover the whole cross-section.

In another literature [6] the 3D effect in EIT was also investigated based on experiment, simulation and analytical models. It was believed that the $3 \mathrm{D}$ attenuation range $(3 / 4$ attenuation) in the axial direction regarding the electrode plane would be one third of the vessel's diameter. It was also concluded that the reconstructed image would move towards the centre of the cross section if the object to be imaged moved away from the electrode plane. These conclusions are applicable to ECT and other ERT sensors. It means that it is difficult to locate and quantify the permittivity or conductivity perturbations inside the electrode plane of ECT or ERT sensors if serious 3D effect exists. Therefore, it is important to reduce the 3D effect of ECT and ERT sensors.

Up to now, few researchers have considered the 3D effect in ECT sensors due to their finite electrode length. Some researchers investigated the $3 \mathrm{D}$ effect by comparing the sensing ranges and the reconstruction errors of different ECT sensors for some distributions [7]. Some researchers tried to determine the optimal electrode length by comparing simulation results of $3 \mathrm{D}$ models with those of $2 \mathrm{D}$ models to make the 3D effect negligible for a certain sensor structure [8]. Some researchers compared resultant capacitance before normalisation from $2 \mathrm{D}$ and $3 \mathrm{D}$ simulations of the same ECT sensor when the sensor had a homogeneous distribution inside, and found that the 3D effect, represented by the difference between absolute capacitance before normalisation from 2D and 3D simulations, would increase almost linearly with permittivity in the image area [9]. All these confirmed that serious 3D effect would occur if an ECT sensor is not properly designed.

In contrast to grounded end guards applied to ECT sensors [7] [8] [9], some researchers [3] [7] [10] used driven guard electrodes below and above measurement electrodes in the axial direction to reduce the $3 \mathrm{D}$ effect. Although some simulation and experimental results seem promising, some other researchers suspected the effectiveness of driven guards [6] [9] [11]. It is necessary to investigate how to reduce the 3D effect efficiently.

Theoretically, to reconstruct a 2D image for ECT or ERT without 3D effect, the electrodes should be infinitely long to produce a uniform field distribution along the axial direction of the sensor. In this case, the 3D field distribution can be simplified to 2D. However, this is impossible for industrial applications. In practice, this can be approximated by taking the following two steps: (1) to make the field distribution as axially uniform as possible within the axial range of the sensor and (2) to confine the field distribution within the axial range of the sensor. Based on previous discussions, the first step can be fulfilled with long measurement electrodes, subject to specific applications. The second step can be fulfilled with grounded end guards since they have been applied to ECT sensors and proven to be effective in narrowing the axial sensing range [7]. From these two points of view, it is necessary to investigate the impact of electrode length and grounded end guards on the 3D effect.

In this paper, the 3D effect of ECT and ERT sensors with variable electrode length is quantified against the corresponding 2D models as references while the quantification process of ERT sensors is preceded by discussions about voltage excitation for ERT. Image reconstruction is implemented based on the previous results to investigate the impact of 3D effect. Grounded guard electrodes are also evaluated to see its effectiveness for reducing the 3D effect. The feasibility of voltage excitation for ERT is verified by experiment. Finally, a common structure is proposed for ECT and ERT sensors to reduce their 3D effect and integrate the two sensors together for dual-modality measurement.

\section{3D EFFECT OF ECT SENSORS}

It was suggested that the optimal length of electrodes in an ECT sensor should be the same as the inner diameter of the pipe for round-shape sensors and the width of pipe for squareshape sensors [8]. However, this conclusion is merely qualitative, without quantitative verification. From the application point of view, it is better to use shorter electrodes to achieve a narrower sensing range in the axial direction. Therefore, it is necessary to investigate the $3 \mathrm{D}$ effect on the normalised capacitance when the electrode length is shorter or much shorter than the diameter of the sensor. It is also necessary to investigate to what extent the normalised capacitance with severe 3D effect would distort the reconstructed images. For those purposes, 3D simulations for ECT sensors with different electrode lengths but without guard electrodes were carried out. Because the frequency of the excitation signal for ECT is in the order of $100 \mathrm{kHz}$ to $1 \mathrm{MHz}$, the corresponding wavelength is larger than the sensor size by several orders of magnitudes. This means the potential distribution inside an ECT sensor can be approximated by the electrostatic field theory. This also applies to ERT sensors. Thus the electrostatic mode in COMSOL Multiphysics is adopted for simulation of ECT sensors and the conductive media DC mode for simulation of ERT sensors. 
The ECT sensor structure and object distribution for simulation are shown in Fig. 1.

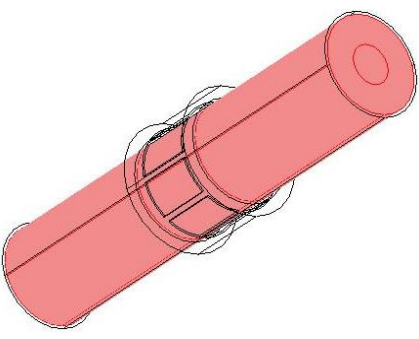

(a) 3D view of ECT sensor without guards

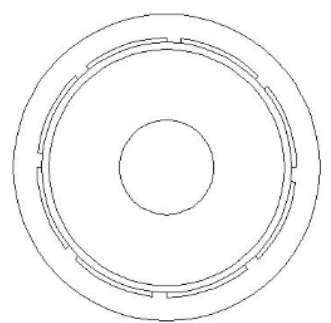

(b) $2 \mathrm{D}$ cross-sectional view of object distribution
Figure 1. ECT sensor structure and object distribution for simulation

Some parameters of the 3D ECT sensor model are:

- Diameter: $10 \mathrm{~cm}$

- Electrode size: $35 \mathrm{~mm} \times 30 \mathrm{~mm} \times 2 \mathrm{~mm}$

- Thickness of pipe wall: $3 \mathrm{~mm}$

- Distance between shield and electrodes: $10 \mathrm{~mm}$

While different lengths of electrodes are used to evaluate the $3 \mathrm{D}$ effect, the length of shield is chosen to be $3 \mathrm{~cm}$ longer than that of electrodes to ensure the validation of the $3 \mathrm{D}$ modeling.

For calibration purpose, the ECT sensor is first filled with air $\left(\varepsilon_{r}=1\right)$ as the lower reference, and then material of relative permittivity 3.0 as the higher reference, i.e. the object to be imaged in Fig .1 (b) has a relative permittivity of 3.0.

By exciting each electrode of the sensor in turn, the resultant electric field distributions when the sensor is filled with air can be obtained by simulation using finite element method (FEM) in COMSOL Multiphysics. Using the postprocessing function in COMSOL Multiphysics, the axial potential distributions for the above ECT sensors with two different electrode lengths, i.e. $1 \mathrm{~cm}$ and $12 \mathrm{~cm}$, are acquired and shown in Fig. 2. Note that different colour represents different potential, e.g. red for $1 \mathrm{~V}$ and dark blue for $0 \mathrm{~V}$. Therefore, Fig. 2 is actually the axial distributions of equipotential lines in different cases.

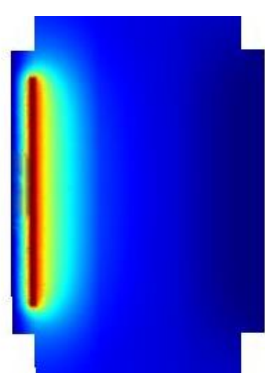

(a) $12 \mathrm{~cm}$

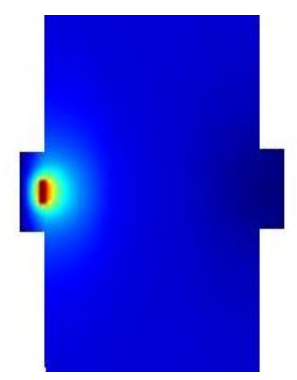

(b) $1 \mathrm{~cm}$
Figure 2. Axial electic field distribution for ECT sensors with two different electode lengths
From Fig. 2, the 3D effect of the ECT sensors with different electrode lengths can be seen qualitatively. It is obvious that long electrodes can generate more uniform axial field distribution (more equi-potential lines are almost in parallel with the axis of the sensor). In this case, the 3D field distribution can be simplified to 2D. This means that an ECT sensor with long electrodes $(12 \mathrm{~cm})$ presents less 3D effect than that with short or pin electrodes $(1 \mathrm{~cm})$. The non-uniform axial field distribution in the case of pin electrodes would cause non-uniform axial sensitivity distribution, because the sensitivity at certain point inside the sensor is closely related to the $3 \mathrm{D}$ electric field distribution generated by exciting one of the electrodes [8]. This non-uniform sensitivity distribution would further cause different responses from the sensor when the same object is placed at different axial positions inside the sensor, similar to that described in [6].

Besides the sensitivity distribution, another essential factor for image reconstruction affected by the $3 \mathrm{D}$ effect is the normalised capacitance because the absolute inter-capacitance between an electrode pair is also related to the electric field distribution. Therefore, the normalised capacitance for 3D ECT sensor models with different electrode lengths is compared with their $2 \mathrm{D}$ reference to see the $3 \mathrm{D}$ effect quantitatively. The normalisation procedure is the same as in [12] and expressed as

$$
\begin{gathered}
\lambda(i, j)=\frac{C_{m}(i, j)-C_{l}(i, j)}{C_{h}(i, j)-C_{l}(i, j)} \\
(i=1 \ldots N, j=i+1 \ldots N)
\end{gathered}
$$

Where $N$ is the number of electrodes, $\lambda(i, j)$ is the normalised capacitance for electrode combination $i-j$, $C_{m}(i, j)$ is the measured capacitance for electrode combination $i-j, C_{l}(i, j)$ and $C_{h}(i, j)$ are the capacitances for electrode combination $i-j$ when the sensor is filled with lower and higher permittivity materials, respectively.

To ensure numerical accuracy (the normalised capacitance differences from a mesh to a finer one are below 2\%) in all the following 3D simulations, the mesh density is increased until the simulated capacitance or conductance after normalisation converges. At the same time, the length of the pipe is varied in different cases until the pipe length has almost no influence on the simulation results. In the simulation, tetrahedral type of elements and direct solver (SPOOLES) are used. The number of elements in each case is the order of 100,000. Take the sensor model shown in Fig. 1 (a) with an electrode length of 12 $\mathrm{cm}$ as an example to show the influence of mesh size on the simulation. The first or coarse mesh is consisted of 66,549 tetrahedral elements, and a finer one consisted of 112,736 elements, which are shown in Fig. 3 (a) and (b) respectively. 
Note that in these two meshes, denser mesh is generated close to the electrodes of the sensor. The simulated capacitance values after normalisation with these two meshes have the maximum difference to be around $1 \%$, as shown in Fig. 4, which displays the first seven values of a whole measurement frame. Note that all the simulations are implemented on a PC with an Intel Core ${ }^{\mathrm{TM}}$ i7-2600 3.4 GHz CPU, 64-bit Windows 7 operation system and 8 GB memory.

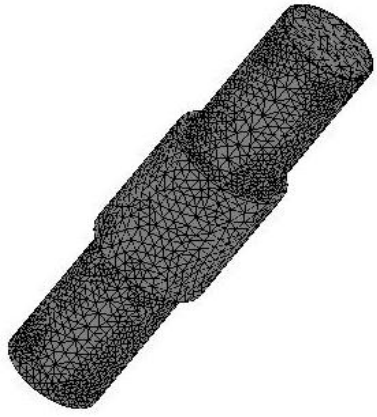

(a) Coarse mesh

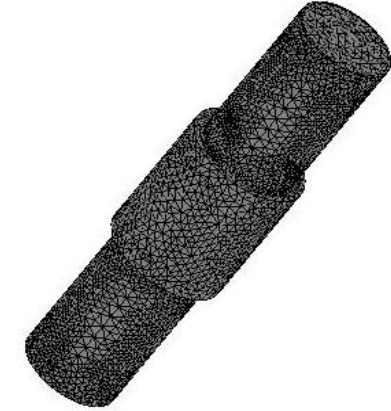

(b) Fine mesh
Figure 3. Comparison between coarse mesh and fine mesh

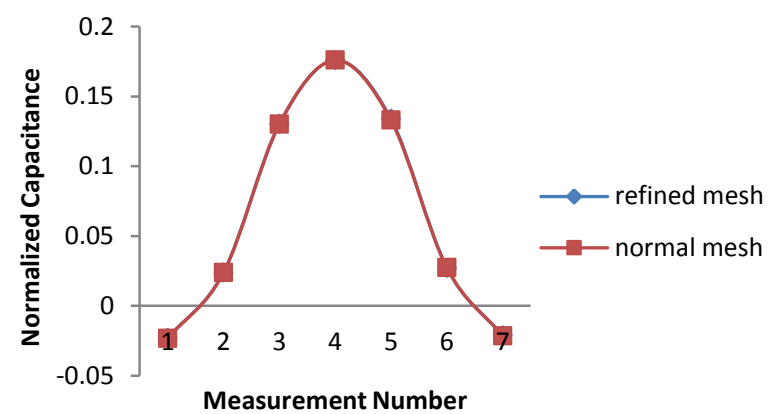

Figure 4. Comparison between normalised capacitances obtained with different mesh sizes

In the simulation, the thickness of electrodes is $2 \mathrm{~mm}$, which is larger than reality (around $1 \mathrm{~mm}$ or less), and it affects the simulation results slightly. This can be verified by comparing the simulated capacitance after normalisation when the thicknesses of electrodes are $2 \mathrm{~mm}$ and $1 \mathrm{~mm}$ respectively. The first seven values in both cases are shown in Fig. 5. The normalised capacitance differences between these two cases are below $2 \%$.

The first seven capacitance values after normalisation of a whole measurement frame for the 3D sensor models are illustrated in Fig. 6. It shows that the normalised capacitance becomes closer and closer to their $2 \mathrm{D}$ reference when the length of electrodes increases. This phenomenon can also be observed from the images reconstructed using the normalised capacitance and the sensitivity maps from the 2D ECT sensor model in Fig. 1 (b) with a uniform distribution of low permittivity material inside. The element in the sensitivity maps, i.e. the sensitivity of electrode pair $\mathrm{i}-\mathrm{j}$ to the permitivity change of the pixel at position $(x, y)$ with an area of $P(x, y)$, can be defined as [13] [14]:

$$
\begin{gathered}
S_{i, j}(x, y)=\int_{P(x, y)} \frac{E_{i}(x, y)}{V_{i}} \cdot \frac{E_{j}(x, y)}{V_{j}} d x d y \\
(i=1 \ldots N, j=i+1 \ldots N)
\end{gathered}
$$

Where $E_{i}(x, y)$ and $E_{j}(x, y)$ are the electric field strengths at $(x, y)$ when the $i_{t h}$ electrode and the $j_{t h}$ electrode function as the excitation electrodes in turn with voltages $V_{i}$ and $V_{j}$ respectively, and $N$ is the number of electrodes.

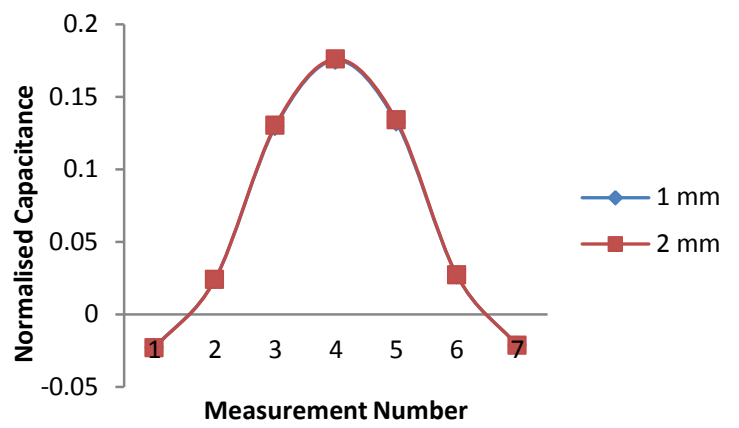

Figure 5. Comparison between normalised capacitances obtained with different electrode thickness

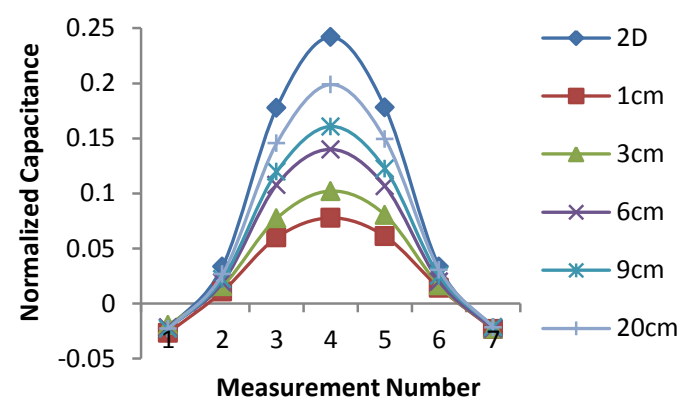

Figure 6. Comparison between normalised capacitance for 2D and 3D ECT sensor models with different electrode lengths and without end guards

The reconstructed images using the Linear BackProjection (LBP) method as described in [15] and expressed as Eq. 3, are shown in Fig. 7.

$$
g=\frac{S^{T} \lambda}{S^{T} \mu_{\lambda}}
$$

Where $g$ is the normalised permittivity vector, $S$ is the sensitivity matrix of the ECT sensor, $S^{T}$ is the transpose of $S$, $\lambda$ is the normalised capacitance vector, and $\mu_{\lambda}$ is the identity vector, i.e. $\mu_{\lambda}=[1,1,1, \ldots, 1] . S$ can be calculated according to Eq. 2 element by element and electrode by electrode, and $\lambda$ can be obtained according to Eq. 1 where the sequence of eletrode combination $i-j$ should comply with that of the calculation process for $S$. 


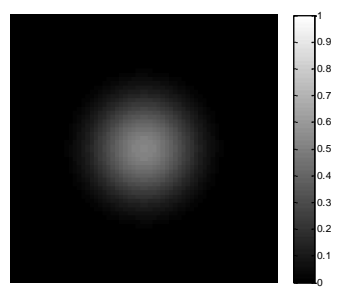

(a) 2D ECT model

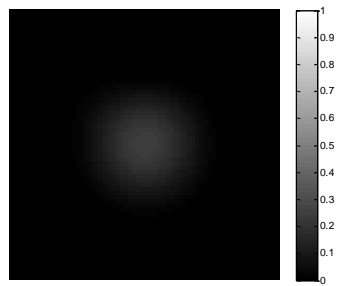

(c) 3D ECT model with $3 \mathrm{~cm}$ electrodes

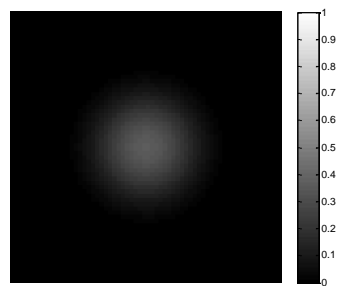

(e) 3D ECT model with $9 \mathrm{~cm}$ electrodes

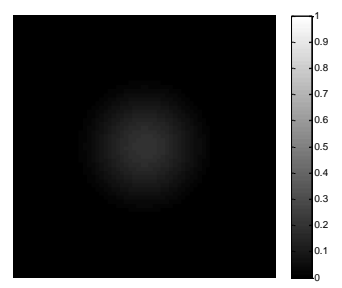

(b) 3D ECT model with $1 \mathrm{~cm}$ electrodes

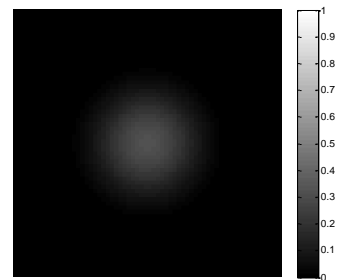

(d) 3D ECT model with $6 \mathrm{~cm}$ electrodes

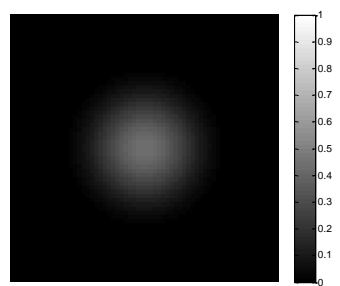

(f) 3D ECT model with $20 \mathrm{~cm}$ electrodes
Figure 7. Image reconstruction results of distribution in Fig. 1 (b) for $2 \mathrm{D}$ and 3D ECT models with different electrode lengths

In Fig. 7, the reconstructed images become more and more similar to the $2 \mathrm{D}$ result when the electrode length increases. To evaluate the image error induced by the 3D effect, the reconstructed image for the 2D ECT sensor model is used as the reference image, and the relative image errors of Fig. 7 (b), (c), (d), (e) and (f) are obtained according to the formula described in [15] and expressed as Eq. 4.

$$
e=\frac{\|\hat{g}-g\|}{\|g\|}
$$

Where $e$ is the relative image error, $g$ is the reference permittivity distribution, $\hat{g}$ is the reconstructed permittivity distribution.

Fig. 8 shows that the relative image errors of the 3D models decrease with the increase in electrode length. This means that the $3 \mathrm{D}$ effect is reduced when longer electrodes are used.

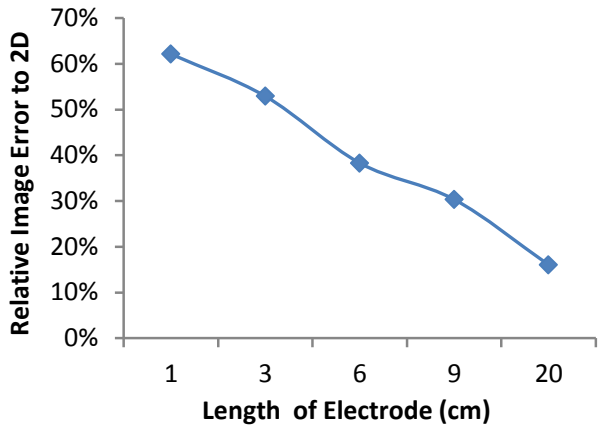

Figure 8. Relative image errors of reconstruction results for 3D ECT models with different electrode lengths regarding to $2 \mathrm{D}$ one

The difference (maximum 25\%) between the 3D normalised capacitance and the $2 \mathrm{D}$ one is still large even when the length of electrodes is twice the inner diameter of the pipe $(10 \mathrm{~cm})$. From this point of view, the 3D normalised capacitance will approach to the $2 \mathrm{D}$ one, only after the electrode length becomes infinite relative to the inner diameter of the pipe, because the electric field distortion still exists on both ends of the sensor and objects outside the sensor also affect the capacitance measurement, even though the uniformity of axial electric field distribution inside the sensor has been improved to a large extent.

In practice, grounded guards are always used at the both ends of an ECT sensor, with which the sensor would have the narrowest sensing range in the axial direction [7]. This would benefit the reduction of $3 \mathrm{D}$ effect. To investigate the $3 \mathrm{D}$ effect of ECT sensors with grounded guards at both ends, simulations were carried out. The 3D simulation model is shown in Fig. 9.

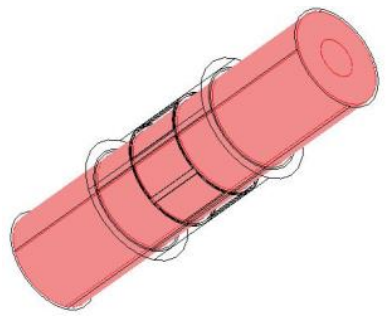

(a) 3D view of ECT sensor with end guards

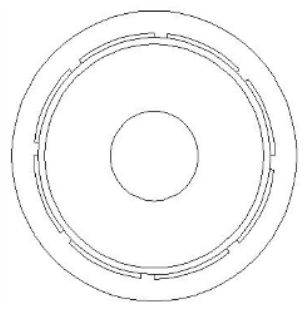

(b) $2 \mathrm{D}$ cross-sectional view of object distribution
Figure 9. 2D and 3D ECT sensor models with grounded end guards

Some parameters of the 3D ECT sensor model with grounded end guards are:

- Thickness of end guards: $2 \mathrm{~mm}$

- Length of end guards: $50 \mathrm{~mm}$

- Gap between end guards and electrodes: $1 \mathrm{~mm}$

Other parameters of this 3D ECT sensor model are the same as the previous one except for the length of shield, which is equal to the sum of electrode length, end guard length and 
the gap between them. The length of end guards is chosen to be half the sensor diameter because Peng et al [8] suggested that this is the optimal length to reduce the 3D effect. Similar simulation and calibration were carried out. For qualitative illustration of the 3D effect in ECT sensors with grounded end guards, the axial electric field distribution for the ECT sensors with $9 \mathrm{~cm}$ electrodes and with or without grounded end guards are shown in Fig. 10 for comparison.

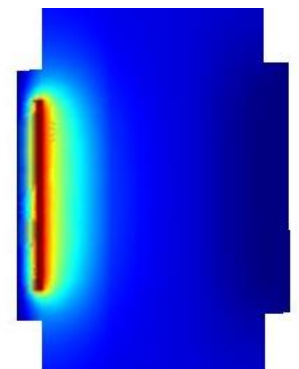

(a) Without end guards

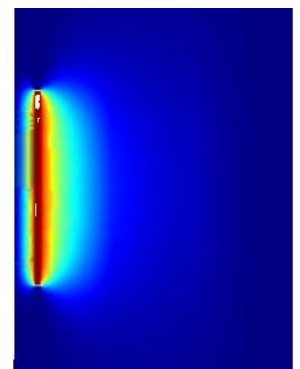

(b) With end guards
Figure 10. Axial electric field distribution for ECT sensors with $9 \mathrm{~cm}$ electrodes and with or without grounded end guards

As mentioned in section I, grounded end guards would help confine the axial sensing range inside the electrode plane, i.e. the axial range of the electric field distortion on both ends of the sensor would be reduced, as shown in Fig. 10. Fig. 10 also shows that the uniformity of axial electric field distribution in this case is changed slightly compared to that for the sensor without end guards. This combination of uniform axial electric field distribution and confined axial sensing range makes it permissible to approximate the 3D electric field distribution within the electrode plane by the corresponding $2 \mathrm{D}$ distribution with less error caused by $3 \mathrm{D}$ effect.

Similar to the previous discussions, the first seven capacitance values after normalisation of a whole measurement frame for the 3D ECT sensor models are obtained and shown in Fig. 11, with comparison to the 2D reference. From Fig. 11, it can be seen that the normalised capacitance for different electrode lengths is closer to the $2 \mathrm{D}$ reference when the grounded guards are used, i.e. the grounded guards can reduce the $3 \mathrm{D}$ effect in the normalised capacitance, even though the electric field would be slightly distorted at both ends of the sensor in this case. Images are reconstructed using the normalised capacitance and the sensitivity maps for the $2 \mathrm{D}$ ECT model. The reconstructed images using LBP are shown in Fig. 12. It shows that the reconstructed images with the $3 \mathrm{D}$ ECT models become more and more similar to the $2 \mathrm{D}$ result as well when the electrode length increases, and each is more similar to the 2D one than the reconstruction with the corresponding 3D ECT sensor without guards, as discussed before.

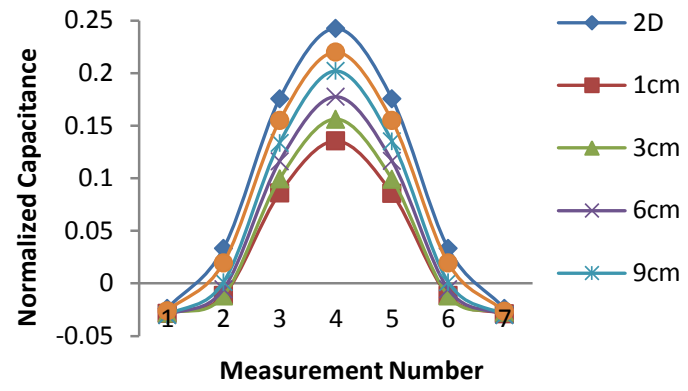

Figure 11. Comparison between normalised capacitance for 2D and 3D ECT sensor models with end guards and different electrode lengths

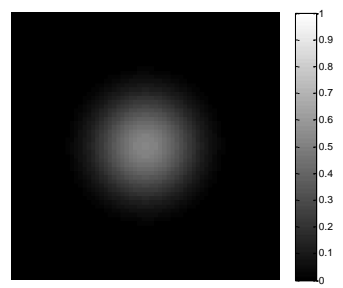

(a) 2D ECT model

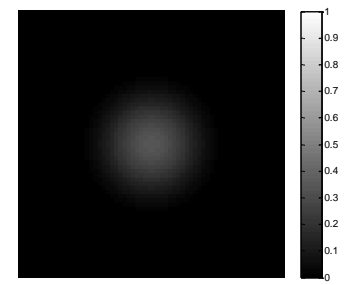

(c) 3D ECT model with $3 \mathrm{~cm}$ electrodes

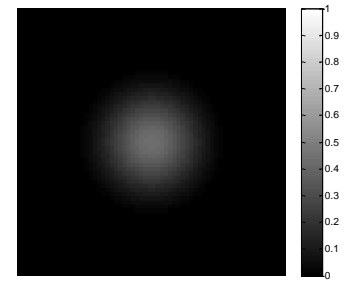

(e) 3D ECT model with $9 \mathrm{~cm}$ electrodes

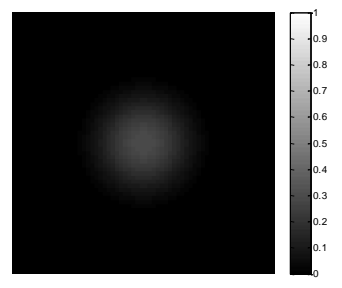

(b) 3D ECT model with $1 \mathrm{~cm}$ electrodes

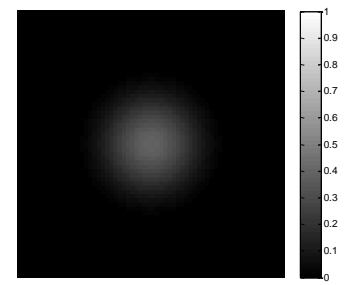

(d) 3D ECT model with $6 \mathrm{~cm}$ electrodes

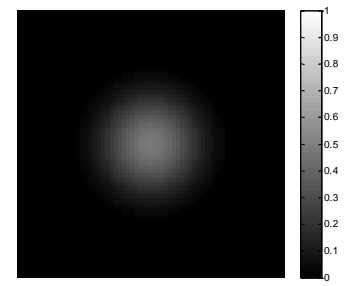

(f) 3D ECT model with $20 \mathrm{~cm}$ electrodes
Figure 12. Image reconstruction results of distribution in Fig. 6 (b) for 2D and 3D ECT models with different electrode lengths and end guards

Similar to the previous part, the reconstructed image with the 2D ECT sensor model is used as the reference image, and the relative image errors of Fig. 12 (b), (c), (d), (e) and (f) are calculated in the same way, which is shown in Fig. 13. From Fig. 13, it can be seen that when grounded end guards are added, the relative image errors for the 3D models are much less than their counterparts in Fig. 8, and also decrease with the increase in the electrode length. This indicates that longer 
electrodes and grounded end guards should be combined together to reduce the $3 \mathrm{D}$ effect in ECT sensor design.

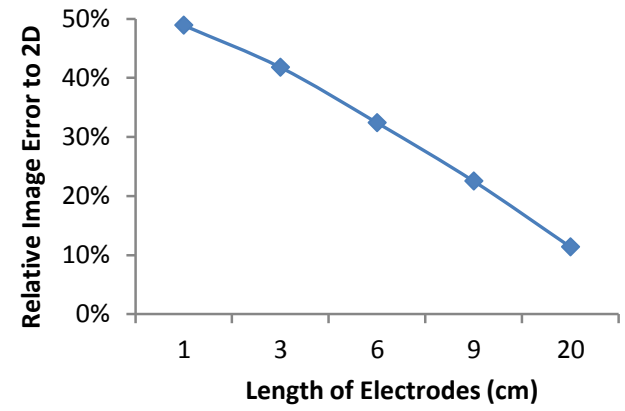

Figure 13. Relative image errors of reconstruction results for 3D ECT models with different electrode lengths and end guards regarding to $2 \mathrm{D}$ one

\section{3D EFFECT OF ERT SENSORS}

A previous paper [16] reported that the field distribution along the axial direction of ERT sensors is extremely inhomogeneous, which leads to the large difference between 2D and 3D simulation results, i.e. large 3D effect. This has also been confirmed by [3] [4], as discussed in Introduction. Therefore, it is very critical to reduce the 3D effect of ERT sensors so that the 3D electric field distribution can be simplified to 2D. To achieve this, two steps are needed: (1) improve the uniformity of electric field distribution along the axial direction of ERT sensors and (2) confine the axial sensing range of the ERT sensors. Similarly, the first can be accomplished by increasing the length of electrodes, and the second can be fulfilled by adding grounded end guards. The reason for the use of grounded end guards in ERT sensors is that the driven guards proposed by some researchers [3] [10] were proved to be less effective by others [6], since there was no evidence that the sensing range of an ERT sensor with driven guards was narrowed compared to normal one without guards. However, the conventional ERT sensor does not allow people to do so. Thus the first part of this section will illustrate the reason and discuss the change needed for ERT sensors to apply the grounded end guards. Then, the effectiveness of long electrodes and grounded end guards on the reduction of the 3D effect in ERT sensors will be discussed.

In the previous section, it has been suggested that grounded end guards can reduce the $3 \mathrm{D}$ effect in an ECT sensor regarding normalised capacitance, but it is not applicable to use this method in ERT sensors when current excitation is adopted because part of the injected current would flow from the injection electrode to the ground directly.
To apply the grounded end guards in an ERT sensor, voltage excitation (similar to that for ECT) can be adopted since the electric fields of ECT and ERT sensors are governed by Laplace equations with exchangeable coefficients and different boundary conditions, which indicates that they are similar to each other if both of ERT and ECT sensors are excited by voltage signals and measured with the same strategy while current injection is commonly used for ERT. Some previous literature [17] [18] claimed the application of voltage excitation in ERT, but the voltage signal was applied to generate their desired current patterns, which means that these systems are still current-excited. Jia et al [19] reported the use of a voltage source and current sensing in an ERT system to output a current with large amplitude (more than $300 \mathrm{~mA}$ ) to highly conductive flows for better measurement accuracy. However, in principle this is still the conventional currentinjection and voltage-measurement strategy.

There are two main reasons for the popularity of current injection for ERT. The first one is the consideration of safety for human body since ERT was originated for detection of diseased human organs the conductance of which is unknown, and the applied current must be within the safety range. For most industrial applications, however, this is no longer the case. The second reason is the influence of contact impedance between the electrodes and human skin or electrolyte on the conductance measurement. This can be ignored in the case of current injection with proper measurement electronics, but much more significant in the case of voltage excitation, as less optimal for ERT measurement compared with current injection [20].

To reduce this influence, there are two possible solutions. The first one is the combination of four-electrode and twoelectrode impedance measurement methods to measure the contact impedance directly, as described in [21]. The second one is the use of capacitively coupled conductivity detection method to avoid direct contact of electrodes and electrolyte and reduce the influence of the contact impedance by parallel or series resonance [22] [23] or by increasing the coupling capacitance between the sensor and the electrolyte [24] [25]. With these solutions, there is a potential that ECT and ERT measurements can be taken with just one common sensor. Similarly, with voltage excitation, there is a potential that ECT and ERT sensors can share a common structure to reduce their $3 \mathrm{D}$ effect, which is another reason why voltage excitation (not current injection) is chosen for ERT. Some experimental results for the verification of ERT by voltage excitation will be presented in section IV.

Based on the above discussion, it is assumed that the influence of the contact impedance can be minimised since 
some very promising results using the solutions listed above have been obtained. As the main purpose of this part is to evaluate the effectiveness of grounded end guards on the reduction of $3 \mathrm{D}$ effect in ERT sensors with voltage excitation, the 3D effect of an ERT sensor without guards is investigated first. The 2D and 3D ERT sensor models without guards are shown in Fig. 14, and each sensor is made up of 12 electrodes. Note that contact impedance is not included in all the sensor models. Some parameters of the 3D ERT sensor model are:

- Diameter: $10 \mathrm{~cm}$

- Electrode size: $70 \mathrm{~mm} \times 18 \mathrm{~mm} \times 2 \mathrm{~mm}$

- Conductive medium inside: Saline with $\sigma=0.02 \mathrm{~S} / \mathrm{m}$

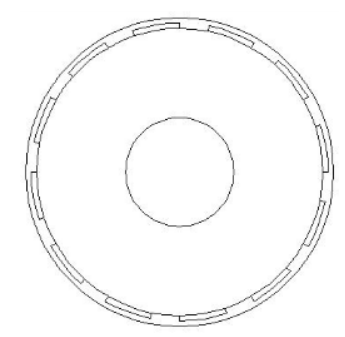

(a) 2D cross-sectional view of object distribution

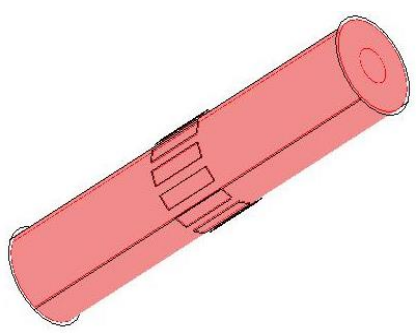

(b) 3D view of ERT sensor without guards
Figure 14. 2D and 3D ERT sensor models

To see the 3D effect, different lengths of electrodes are used. Similar to ECT, each time one electrode is applied a voltage signal and all the other electrodes are grounded for measuring currents through the medium. This process is repeated until all the independent measurements are taken. Because the voltage-excitation and current-measurement method is used for conductance measurement, the exact same calibration or normalisation procedure as ECT can be adopted to reduce $3 \mathrm{D}$ effect contained in measurements. Also, sensitivity maps for ERT sensors are generated in a similar way to that for ECT sensors. Since the object to be imaged in Fig. 14 (a) is non-conductive $(\sigma=0 S / m)$, the lower reference for conductance is 0 . The higher reference is acquired when the simulation model is filled with homogeneous saline listed above, and then normalisation process is taken after the measurement data are obtained. The first eleven values of normalised conductance with 2D and 3D ERT sensor models with different electrode lengths and without end guards are shown in Fig. 15.

Similarly, the normalised conductance with the 3D ERT models would become closer and closer to that with the 2D model when the electrode length increases. Based on the normalised conductance of a whole measurement frame for each 3D ERT sensor and sensitivity maps of the 2D ERT sensor model generated in the same way as in ECT, images are reconstructed for the distribution in Fig .14 (a) using LBP, and the results are shown in Fig. 16. With the reconstruction result with the $2 \mathrm{D}$ sensor model as the reference image, the relative image errors of Fig. 16 (b), (c), (d), (e) and (f) are calculated as shown in Fig. 17.

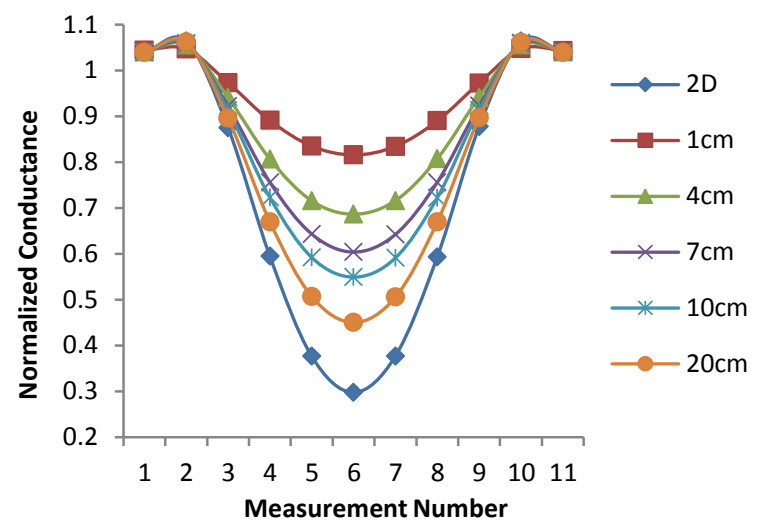

Figure 15. Comparison between normalised conductance for 2D and 3D ERT sensor models with different electrode lengths and without end guards

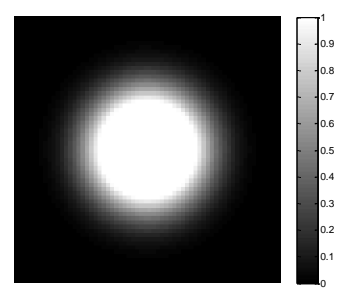

(a) 2D ERT model

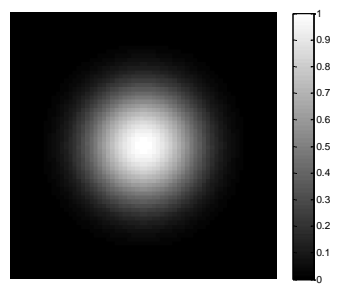

(c) 3D ERT model with $4 \mathrm{~cm}$ electrodes

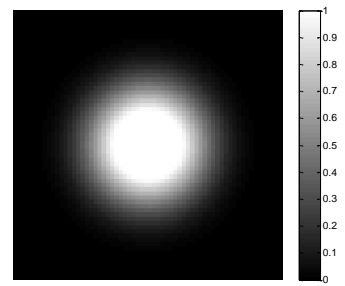

(e) 3D ERT model with $10 \mathrm{~cm}$ electrodes

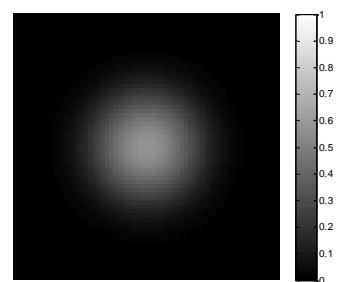

(b) 3D ERT model with $1 \mathrm{~cm}$ electrodes

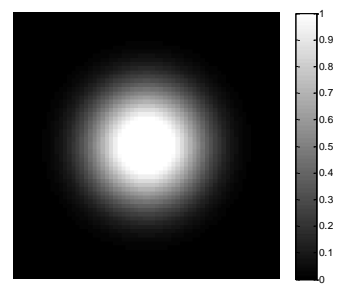

(d) 3D ERT model with $7 \mathrm{~cm}$ electrodes

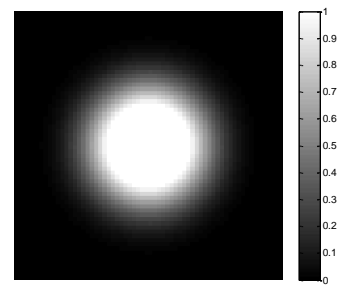

(f) 3D ERT model with $20 \mathrm{~cm}$ electrodes
Figure 16. Image reconstruction results of distribution in Fig. 10 (a) for 2D and 3D ERT models with different electrode lengths and without end guards 


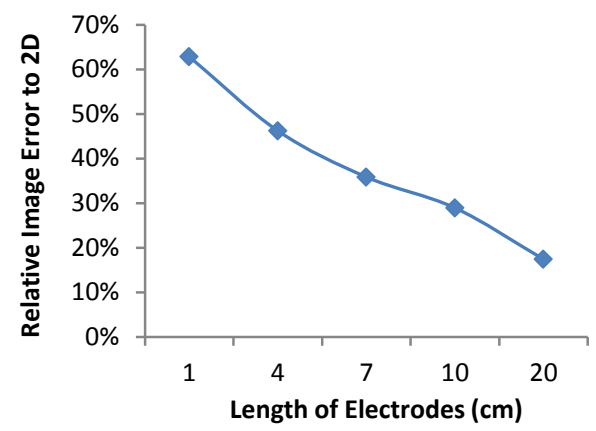

Figure 17. Relative image errors of reconstruction results for 3D ERT models with different electrode lengths and without end guards regarding to $2 \mathrm{D}$ one

As expected, the relative image errors for the 3D ERT sensor models decrease with the increase in electrode length, which is similar to the corresponding results for the 3D ECT sensors. This also confirms that the electric fields of ECT and ERT sensors are similar to each other when both are excited in the same way and similar 3D effect exists in both of them.

To compare with the above results, grounded end guards are added to the above 3D ERT sensors, and the 2D and 3D sensor structures are shown in Fig. 18. Some parameters of the 3D ERT sensor model with grounded end guards are:

- Thickness of end guards: $2 \mathrm{~mm}$

- Length of end guards: $50 \mathrm{~mm}$

- Gap between end guards and electrodes: $5 \mathrm{~mm}$

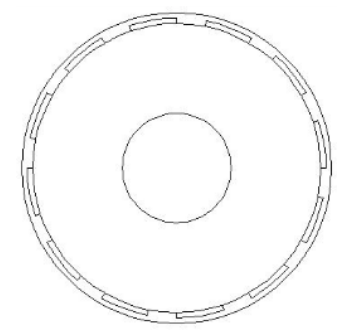

(a) 2D cross-sectional view of object distribution

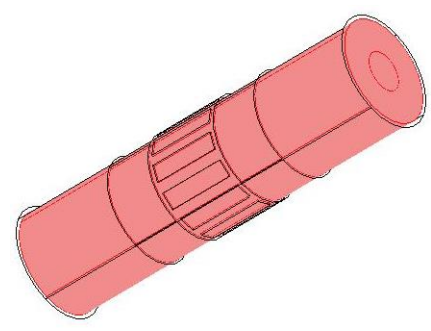

(b) 3D view of ERT sensor with end guards
Figure 18. 2D and 3D ERT sensor models with grounded end guards

Other parameters are the same as the ERT sensors without guards. Note that unlike ECT sensors, no shield is included in the ERT sensor structure since no conductive medium exists between shield and pipe wall and the simulation results would be the same with or without the shield. Similar simulations and normalisations are carried out and the first eleven values of normalised conductance for 2D and 3D ERT sensor models with different electrode lengths and grounded end guards are shown in Fig. 19. The corresponding reconstruction results using LBP are shown in Fig. 20, while the relative image errors for the 3D ERT models with different electrode lengths and grounded end guards are shown in Fig. 21, referred to the reconstructed image for the $2 \mathrm{D}$ model.

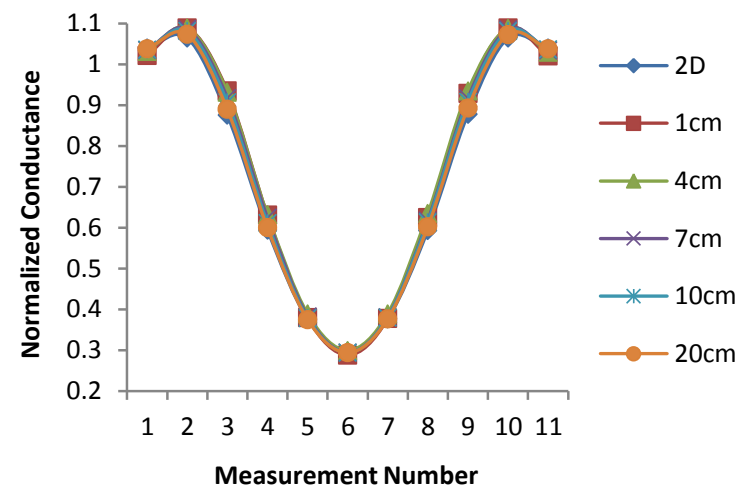

Figure 19. Comparison between normalised conductance for 2D and 3D ERT sensor models with end guards and different electrode lengths (a) 2D ERT model

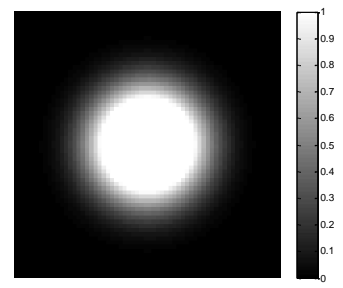

(c) 3D ERT model with $4 \mathrm{~cm}$ electrodes

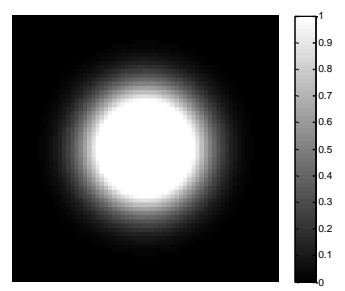

(e) 3D ERT model with $10 \mathrm{~cm}$ electrodes

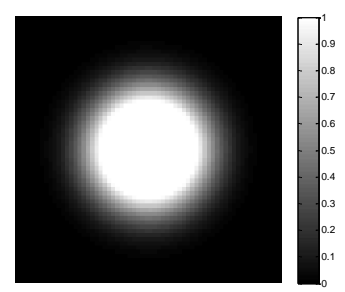

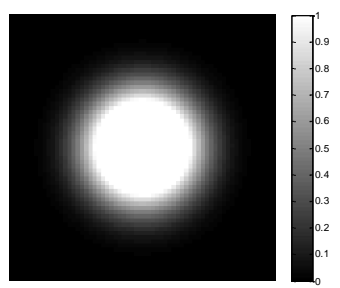

(b) 3D ERT model with $1 \mathrm{~cm}$ electrodes

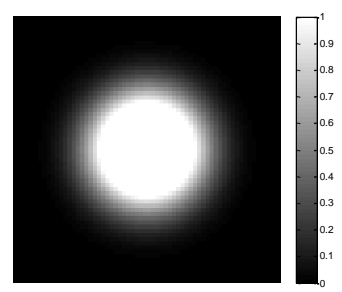

(d) 3D ERT model with $7 \mathrm{~cm}$ electrodes

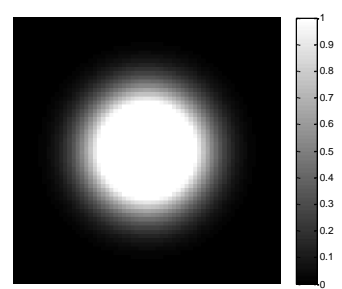

(f) 3D ERT model with $20 \mathrm{~cm}$ electrodes
Figure 20. Image reconstruction results of distribution in Fig. 14 (a) for 2D and 3D ERT models with different electrode lengths and end guards

Strangely, the relative image errors for all 3D ERT sensor models with end guards are well below $6 \%$ regardless of the electrode length in Fig. 21, even though the relative image error of the 3D ERT sensor model with electrode length of 4 $\mathrm{cm}$ is slightly larger than the 3D sensor model with electrode length of $1 \mathrm{~cm}$. This is very different from the results for 3D 
ECT sensors with end guards in Fig. 13. The possible reason for this phenomenon is that the non-conductive object being imaged is placed along the middle axis of the pipe and causes the current to flow around the pipe wall and the grounded end guards, which are mounted on the inner surface of the pipe wall and absorb almost all the current that tends to flow outside the sensor plane. However, in ECT the contrast of high permittivity to low permittivity is only $3: 1$. A significant part of the electric field lines can still penetrate the high permittivity material and spread along the middle axis of the pipe with only a few of them drawn to the grounded end guards. This is verified by changing the conductivity of the object to be imaged in Fig. 18 (a) from $\sigma=0 \mathrm{~S} / \mathrm{m}$ to be $\sigma=0.06 \mathrm{~S} / \mathrm{m}$, with which the conductivity contrast of the object to the background is $3: 1$ the same as the permittivity contrast in ECT sensor simulations. Note that both the nonzero lower reference and higher reference need to be acquired in this case for normalisation of measured conductance. The first eleven values of normalised conductance in this case are shown in Fig. 22

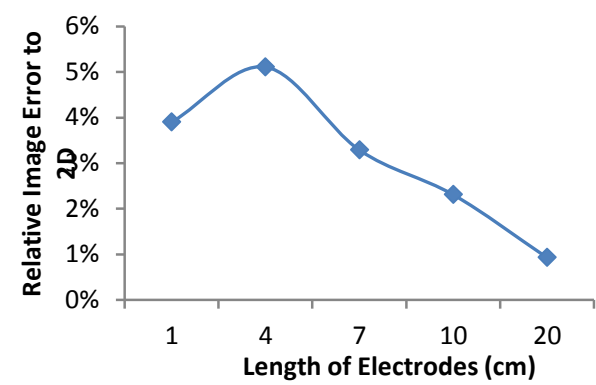

Figure 21. Relative image errors of reconstruction results for 3D ECT models with different electrode lengths and end guards regarding to $2 \mathrm{D}$ one

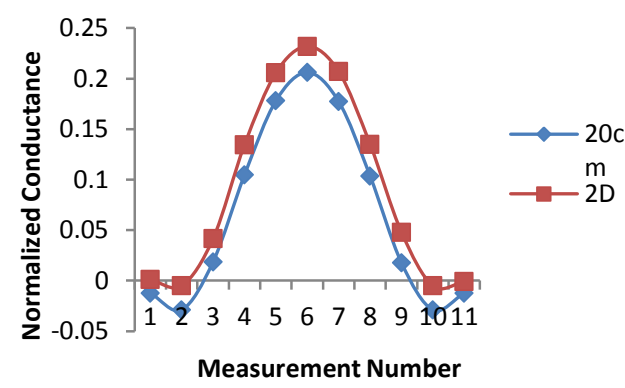

Figure 22. Comparison between normalised conductance for 2D and 3D ERT sensor models with electrode length of $20 \mathrm{~cm}$ and end guards when object being imaged is conductive

The comparison result shown in Fig. 22 is similar to that for the ECT sensor in Fig. 11, conforming the explanation above. The conclusion is that similar 3D effect exists in ECT and ERT sensors with the same permittivity or conductivity contrast of object to background. Since there is much larger difference between normalised conductance for 2D and 3D ERT sensors with electrode length of $20 \mathrm{~cm}$ in Fig. 22 than in Fig. 19, normalised conductance for other 3D ERT sensors with electrode lengths less than $20 \mathrm{~cm}$ should have even larger difference than those in Fig. 19, as indicated by previous simulation results.

\section{EXPERIMENTAL VERIFICATION OF ERT BY VOLTAGE EXCITATION}

To validate the ERT sensor design with voltage excitation, experiments were carried out using an impedance-analyserbased multi-channel imaging system. The performance and detailed information about this imaging system can be found in [26]. The system setup is shown in Fig. 23. For this feasibility study, an available ECT sensor was used for conductance measurement. As shown in Fig. 24 (a), the ECT sensor has 8 large electrodes mounted on the inner surface of the tube, which directly contact with the electrolyte inside the sensor. Its $2 \mathrm{D}$ simulation model is shown in Fig. 24 (b). These large electrodes can help reduce the influence of contact impedance between electrodes and electrolyte on the conductance measurement, but the contact impedance is not measured and calibrated. Some parameters of the ECT sensor are listed below:

- Diameter: $7.4 \mathrm{~cm}$

- Electrode size: $65 \mathrm{~mm} \times 25 \mathrm{~mm} \times 1 \mathrm{~mm}$

- Thickness of pipe wall: $3 \mathrm{~mm}$

- Distance between shield and electrodes: $11 \mathrm{~mm}$

- Height of the tube and shield: $17.5 \mathrm{~cm}$

- Length of grounded end guards: $2 \mathrm{~cm}$

- Gap between the end guards and electrodes: $5 \mathrm{~mm}$

- Thickness of tube bottom: $3 \mathrm{~cm}$

- Distance between the tube bottom and bottom end guards: $1 \mathrm{~cm}$

- Conductive medium inside: Saline $(\sigma=1.045 \mathrm{mS} /$ $\mathrm{cm})$.

With an impedance analyzer HP4192, an ac voltage of 1.1 $\mathrm{V}$ is applied to one electrode of the ECT sensor and other electrodes are grounded for conductance measurement one by one. Different excitation frequencies, $100 \mathrm{kHz}, 1 \mathrm{MHz}$ and 10 $\mathrm{MHz}$, were chosen to see whether the frequency has significant impact on the experimental results. 2D simulations based on the setup shown in Fig. 24 (b) are also conducted under the mode of In-Plane Electric Currents (Quasi-statics, electric) in COMSOL Multiphysics. Because the contact impedance is not considered in simulations, the normalised conductance at the above three frequencies for the 2D simulation setup are almost the same (below $2 \%$ difference). Thus only the 2D simulation results at the excitation frequency of $100 \mathrm{kHz}$ is shown in Fig. 25 together with the experimental results at those three 
excitation frequencies. Note that the measured and simulated conductance are normalised in the same way as in section III.

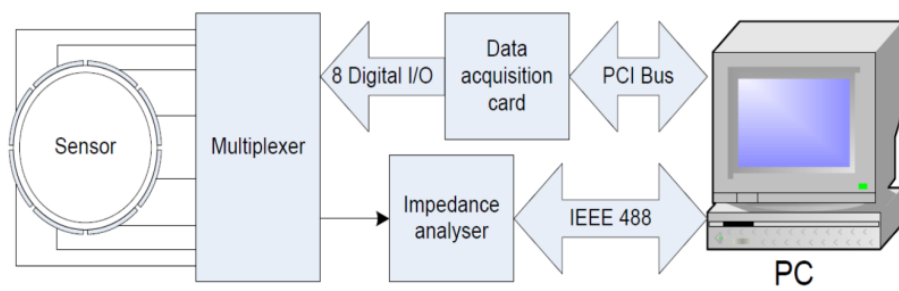

Figure 23. Experimental system setup for verification of ERT with voltage excitation

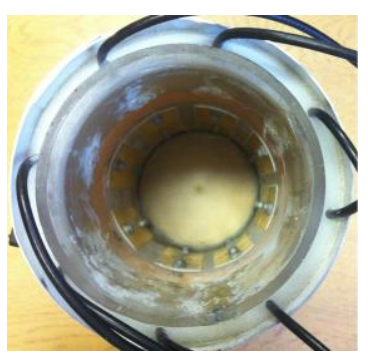

(a) 3D view

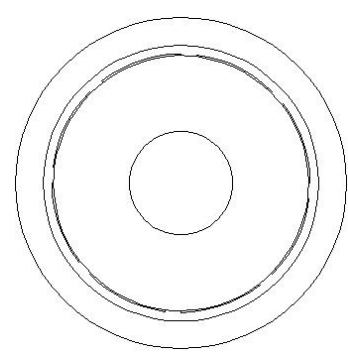

(b) 2D simulation model
Figure 24. 3D view of and 2D simulation model for ERT sensor used in experiments

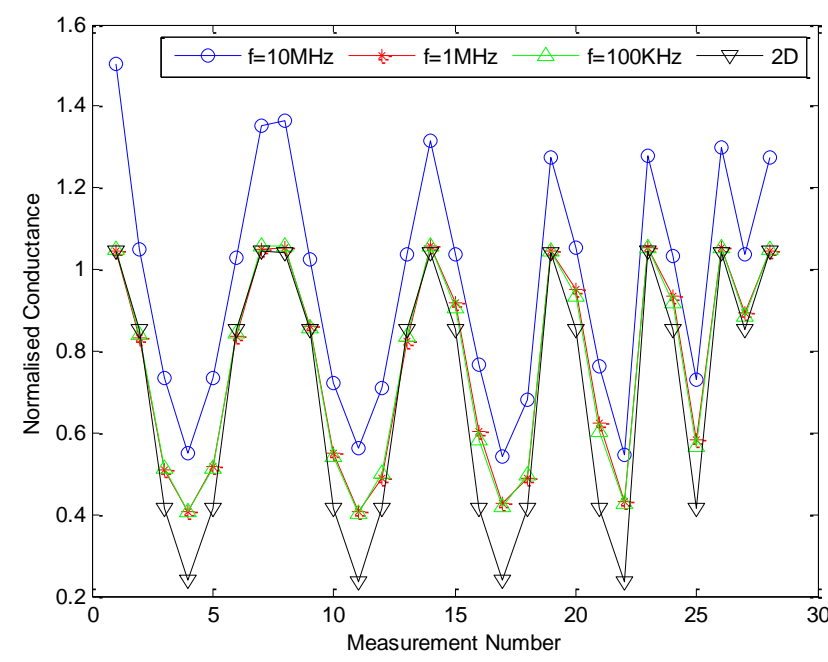

Figure 25. Comparison between normalised conductance from 2D simulation and experimental setup at different excitation frequencies

Apparently, the normalised conductance values at frequencies of $1 \mathrm{MHz}$ and $100 \mathrm{kHz}$ are close to each other, but large difference exists between these two and that at frequency of $10 \mathrm{MHz}$, perhaps because of the contact impedance, which was claimed to be equivalent to a combination of a resistive part and a capacitive part [27] [28] [29]. While the impedance value of the capacitive part depends on the excitation frequency, $10 \mathrm{MHz}$ signal would decrease this value to a comparable level with the impedance value of the resistive part but the values with $100 \mathrm{kHz}$ and $1 \mathrm{MHz}$ signals are much larger. This decrease would enable more current to be injected into the medium inside the sensor and redistribution of the current through each detection electrodes when one electrode is excited with an ac voltage signal of fixed amplitude. The contact impedance also contributes to the difference between the $2 \mathrm{D}$ result and experimental result because it constitutes part of the measured resistance or conductance from experiments.

Another reason for this difference is the 3D effect. Even though their length is $2 \mathrm{~cm}$ (a little more than $1 / 4$ of the sensor diameter), the end guards in the ERT sensor used in experiments are mounted on the outer surface of the tube wall, which has no effect on confining the axial sensing range of the ERT sensor (no contact with electrolyte). Therefore, the current can flow outside the sensor plane and then return to the detection electrodes, but it cannot in the 2D simulation. This shuttle effect increases the conductance between the detection electrode and the excitation electrode, especially when the detection electrode is opposite to the excitation electrode. However in previous simulations for the ERT sensor with grounded end guards, the guards length is half the sensor diameter as suggested by [8], and the guards directly contact with the electrolyte, which help reduce the 3D effect effectively as shown in Fig. 19. Other reasons for the asymmetric distribution of normalised conductance with measurement number in Fig. 25 would be the fabrication error of the sensor and the measurement noise.

With these normalised conductance from 2D simulation and experiment setup at different excitation frequencies, the object distribution in Fig. 24 (a) can be reconstructed using LBP, as shown in Fig. 26. Note that the sensitivity maps are generated from the above $2 \mathrm{D}$ simulation model in the same way as in section II. Fig. 26 shows that the correct position and shape of the object can be reconstructed at different excitation frequencies, even though image artifacts exist. This can partially verify the feasibility of the ERT sensor with voltage-excitation and current- measurement. If the contact impedance of the above ERT sensor can be measured and calibrated, then the reconstructed images in Fig. 26 (a), (b) and (c) can be closer to their $2 \mathrm{D}$ reference. 


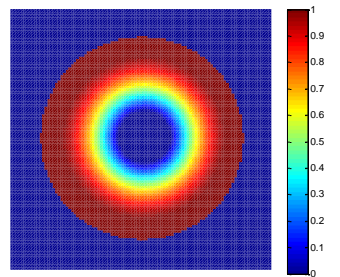

(a) $100 \mathrm{kHz}$

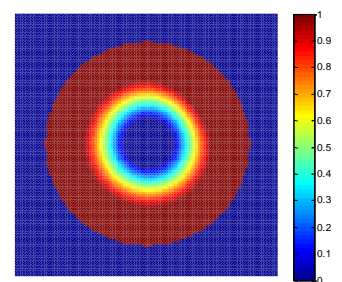

(c) $10 \mathrm{MHz}$

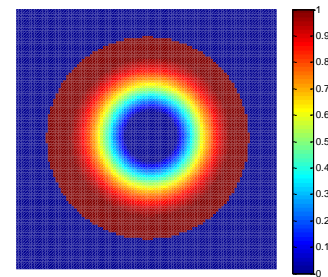

(b) $1 \mathrm{MHz}$

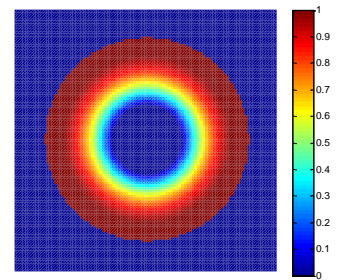

(d) 2D reference
Figure 26. Image reconstruction results of distribution in Fig. (a) for 2D ERT simulation model and experimental setup with different excitation frequencies

\section{COMMON STRUCTURE FOR ECT AND ERT SENSORS}

As discussed in section II and III, grounded end guards can be applied to both ECT and ERT sensors to reduce their 3D effect, if voltage excitation is adopted for ERT. With voltage excitation for ERT, only additional calibrations or measurements need to be taken to remove the influence of contact impedance on the conductance measurement. From this point of view, ECT and ERT sensors can share a common structure similar to that in Fig. 9 (a) to narrow their sensing ranges in the axial direction, but the electrodes should be mounted on the inner or outer surface of the pipe wall, depending on the choice of the method for reducing the influence of contact impedance as discussed in section III. This is not only beneficial for reduction of $3 \mathrm{D}$ effect, but also for dual-modality measurement needed in some industrial processes. Li and Yang [2] discussed the necessity and development of multi-modality tomography systems before 2009, and did some initial simulations and experiments to investigate the feasibility of ECT and ERT dual-modality system with voltage excitation, and some promising results were obtained. This also confirms that an ERT system with voltage excitation is feasible. However, they used two separate sensors for ECT and ERT measurements. Marashdeh et al [30] proposed a multi-modal based on ECT sensors, which used capacitance measurements for permittivity imaging and power balance measurements for conductivity imaging. Even though it seems promising, this kind of systems suffers from nonlinear errors and skin depth effect with high conductivity. Also, the proposed reconstruction technique is only valid when the capacitance signal can be assumed to be independent from the power signal, which is difficult to be fulfilled in most industrial applications.

To avoid these drawbacks and reduce the $3 \mathrm{D}$ effect, the approach described in session III, with which only one sensor is needed, is suggested for both ECT and ERT measurements while related electronics can be designed in the same way as proposed in [2]. With this structure, the sensor design for ECT and ERT dual-modality measurement would be simplified [30], and the interference between two separate sensors or two measurement signals can be eliminated. More temporally closely correlated and independent ECT and ERT measurements can also be acquired at the same time, which is beneficial for dynamic measurements required in some industry processes.

\section{CONCLUSIONS}

Similar 3D effect exists in both ECT and ERT sensors. The 3D distribution of electric field would distort the shape and displace the positions of objects being imaged in a reconstructed $2 \mathrm{D}$ image. By comparing the simulation results of the 3D ECT and ERT sensor models after normalisation with the corresponding $2 \mathrm{D}$ one, it is concluded that long electrodes can improve the uniformity of axial electric field distribution and reduce the 3D effect in ECT or ERT sensors. The negative effect is that it would enlarge the sensing range. To solve this problem, grounded end guards are commonly used in ECT sensors. Simulation suggested that grounded end guards can reduce the $3 \mathrm{D}$ effect contained in capacitance after normalisation, but it cannot be used in ERT sensors when current injection is adopted. To apply grounded end guards in ERT sensors, the voltage-excitation and current-measurement strategy is first introduced into ERT measurements, and validated by experiments. Simulation results indicate that grounded end guards can also reduce the $3 \mathrm{D}$ effect in ERT sensors.

Images are reconstructed for a centered object in ECT and ERT sensors without or with end guards using LBP. It is shown that reconstructed images for the $3 \mathrm{D}$ sensor models approach to the corresponding 2D result when the electrode length increases or after grounded end guards are added in both cases. Finally, a common structure is proposed for ECT and ERT sensors to reduce their $3 \mathrm{D}$ effect, which also has potential applications for dual-modality measurements in industrial processes. 


\section{ACKNOWLEDGEMENT}

Jiangtao Sun would like to thank the Chinese Scholarship Council for financially supporting his $\mathrm{PhD}$ study at The University of Manchester.

\section{REFERENCES}

[1] F. Dickin, and M. Wang, "Electrical resistance tomography for process applications,” Meas. Sci. Technol., vol. 7, pp. 247-260, 1996

[2] Y. Li, and W.Q. Yang, "Measurement of multi-phase distribution using an integrated dual-modality sensor," Proc. IEEE International Workshop on Imaging Systems and Techniques, Shenzhen, China, May 2009, pp. 335-339.

[3] Y.X. Ma, H. Wang, L.A. Xu, and C.Z. Jiang, "Simulation study of the electrode array used in an ERT system," Chemical Engineering Science, vol. 52, pp. 2197-2203, 1997.

[4] E. Fransolet, M. Crine, G.L. Homme, D. Toye, and P. Marchot, "Electrical resistance tomography sensor simulations: comparison with experiments,” Meas. Sci. Technol., vol. 13, pp. 1239-1247, 2002.

[5] K. S. Rabbani, and A. M. B. H. Kabir, "Studies on the effect of the third dimension on a two-dimensional electrical impedance tomography system," Clin. Phys. Physiol. Meas., vol. 12, pp. 393-402, 1991.

[6] M. Wang, "Three-dimensional Effects in Electrical Impedance Tomography," Proc. $1^{\text {th }}$ World Congress on Industrial Process Tomography, Boxton, United Kindom, April 1999, pp. 410-415.

[7] H. Yan, F.Q. Shao, H. Xu, and S. Wang, "Three-dimensional analysis of electrical capacitance tomography sensing fields," Meas. Sci. Technol., vol. 10, pp. 717-725, 1999.

[8] L.H. Peng, C.H. Mou, D.Y. Yao, B.F. Zhang, and D.Y. Xiao, "Determination of the optimal axial length of the electrode in an electrical capacitance tomography sensor," Flow Measurement and Instrumentation, vol. 16, pp. 169-175, 2005.

[9] K.J. Alme, and S. Mylvaganam, "Electrical Capacitance TomographySensor Models, Design, Simulations, and Experimental Verification," IEEE SENSORS JOURNAL, vol. 6, pp. 1256-1266, 2006.

[10] Y.X. Ma, L.A. Xu, and C.Z. Jiang, "Experimental study of the guard electrodes in an ERT system," Proc. $1^{\text {th }}$ World Congress on Industrial Process Tomography, Boxton, United Kindom, April 1999, pp. 335-338.

[11] W.Q Yang, "Design of electrical capacitance tomography sensors," Meas. Sci. Technol., vol. 21, pp. 1-13, 2010.

[12] C.G. Xie, S.M. Huang, B.S. Hoyle, R. Thorn, C. Lenn, D. Snowden, and M.S. Beck, "Electrical capacitance tomography for flow imaging: system model for development of image reconstruction algorithms and design of primary sensors,” IEE Proc.-G, vol. 139, pp. 89-98, 1992.

[13] W.R.B. Lionheart, "Reconstruction Algorithms for Permittivity and Conductivity Imaging," Proc. of the 2nd World Congress on Industrial Process Tomography, Hannover, Germany, August 2001, pp. 4-11.

[14] S. Liu, W.Q. Yang, H. Wang, F. Jiang, and Y. Su, "Investigation of square fluidized beds using capacitance tomography: preliminary results,” Meas. Sci. Technol., vol. 12, pp. 1120-1125, 2001
[15] W.Q. Yang and L.H. Peng, "Image reconstrcution algorithms for electrical capacitance tomography," Meas. Sci. Technol., vol. 14, pp. R1-R13, 2003.

[16] J.T. Sun, and W.Q. Yang, "3D issues of electrical capacitance and resistance tomography sensors," Proc. IASTED Int. Conf. on Signal and Image Processing and Applications, Crete, Greece, June 2011.

[17] A. Hartov, E. Demidenko, N. Soni, M. Markova and K. Paulsen, "Using voltage sources as curent drivers for electrical impedance tomography," Meas. Sci. Technol., vol. 13, pp. 1425-1430, 2002.

[18] F.J. Lidgey, Q.S. Zhu, C.N. McLeod and W.R. Breckon, "Electrode current determination from programmable voltage souces," Clin. Phys. Physiol. Meas., vol. 13, pp. 43-46, 1992.

[19] J.B. Jia, M. Wang, H.I. Schlaberg and H. Li, "A novel tomographic sensing system for high electrically conductive multiphase flow measurement," Flow Measurement and Instrumentation, vol. 21, pp. 184-190, 2010.

[20] G.J. Saulnier, A.S. Ross and N. Liu, "A high-precision voltage souce for EIT,” Physiol. Meas., vol. 27, pp. S221-S236, 2006.

[21] R. Cardu, P.H.W. Leong, C.T. Jin and A. McEwan, "Electrode contact impedance sensitivity to variations in geometry," Physiol. Meas., vol. 33, pp. 817-830, 2012.

[22] C.Y. Shih, W. Li, S.Y. Zheng, and Y.C. Tai, "A resonance-induced resolution enhancement method for conductivity sensors," Proc. of 5th IEEE Conf. on Sensors, EXCO, Daegu, Korea, October 2006, pp. 271274

[23] Z.Y. Huang, W.W. Jiang, X.M. Zhou, B.L. Wang, H.F. Ji and H.Q. Li, "A new method of capacitance coupled contactless conductivity detection based on series resonance," Sensors and Actuators B: Chemical, vol. 143, pp. 239-245, 2009

[24] B.L. Wang, Z.Y. Huang, H.F. Ji and H.Q. Li, "Towards Capacitively Coupled Electrical Resistance Tomography," Proc. of 6th World Congress on Industrial Process Tomography, Beijing, China, September 2010, pp. 1574-1577.

[25] R. Hayes, F. Podd, P. Newill, B.D. Grieve and T.A. York, "Capacitively-coupled impedance measurements for ERT," Proc. of 6th International Symposium on Process Tomography, Cape Town, South Africa, March 2012.

[26] X.H. Hu, M. Yang, I. Ismail, Y. Li, W.Q. Yang and M. Manrique de Lara, "An impedance-analyser-based multi-channel imaging system and its applications," Proc. IEEE International Workshop on Imaging Systems and Techniques, Chania, Greece, September 2008, pp. 181-186.

[27] J.G. Webster, Medical Instrumentation: Application and Design, 3rd ed.. New York: Wiley, 1998

[28] E.T. McAdams, J. Jossinet, A. Lackermeier and F. Risacher, "Factors affecting electrode gel-skin interface impedance in electrical impedance tomography,” Med. Biol. Eng. Comput., vol. 34, pp. 397-408, 1996.

[29] A. Boyle and A. Adler, "The impact of electrode area, contact impedance and boundary shape on EIT images," Physiol. Meas., vol. 32, pp. 745-754, 2011.

[30] Q. Marashdeh, W. Warsito, L.S. Fan and F.L. Teixeira, "A Multimodal Tomography System Based on ECT Sensors," IEEE SENSORS JOURNAL, vol. 7, pp. 426-433, 2007. 